\title{
TEORÍAS IMPLÍCITAS DEL LIDERAZGO MASCULINO Y FEMENINO SEGÚN ÁMBITO DE DESEMPEÑO
}

\section{IMPLICIT LEADERSHIP THEORIES ABOUT MASCULINE AND FEMININE LEADERSHIP ACCORDING WORK CONTEXT}

\author{
María Laura Lupano Perugini \\ Consejo Nacional de Investigaciones Científicas y Técnicas, Argentina \\ Universidad de Palermo, Argentina \\ Alejandro Castro Solano \\ Consejo Nacional de Investigaciones Científicas y Técnicas, Argentina \\ Universidad de Palermo, Argentina
}

\begin{abstract}
Resumen: Se intentó analizar si existen diferencias en las Teorías Implícitas del Liderazgo (TILs) acerca del liderazgo masculino y femenino según ámbito de desempeño (empresarial, académico y político). Además se buscaron diferencias según ámbito y según sexo de quien responde, para el caso de las TILs acerca del liderazgo femenino. Participaron 441 sujetos (50,3\% varones; 49,7\% mujeres). Se diseñó un instrumento ad-hoc que solicitaba caracterizar a un/una líder efectivo/a de acuerdo al ámbito. Los resultados muestran que no existen diferencias en las TILs acerca del liderazgo masculino y femenino según ámbito, pero si existen diferencias por ámbitos tomando las TILs en conjunto. Además se hallaron diferencias en las TILs acerca del liderazgo femenino según ámbito y según sexo de quien responde.
\end{abstract}

Palabras clave: liderazgo, teorías implícitas, género, ámbito.

\begin{abstract}
Research was conducted to find if exist differences in implicit leadership theories (ILTs) about masculine and feminine leadership according work context (enterprise, academic and political). Besides, we look for differences in feminine ILTs according work context, and sex of the participant. Participate 441 adults (50,3\% male; 49,7\% female). The data collection was realized by a survey designed ad-hoc, in which the participants were requested to describe a male/ female leader according work context. The obtained results do not evidence differences in masculine and feminine ILTs according work context, but show differences in ILTs considered globally. Besides we found differences in feminine ILTs according work context, and sex of the participant.
\end{abstract}

Keywords: leadership, implicit theories, gender, context.

\section{MUJERES Y LIDERAZGO}

Hombres y mujeres difieren en el acceso a puestos de liderazgo. Esto se refleja en el hecho de que una de las desigualdades más llamativas presentes en la mayoría de las sociedades occidentales es la menor presencia de mujeres en puestos de dirección en distintos ámbitos y organizaciones (Cuadrado, 2004). La diferencia a favor de los hombres en dicho acceso se la ha explicado a través de la metáfora del Techo de Cristal (Federal Glass Ceiling Commission, 1995; Morrison, White
\& Van Velsor, 1987) que intenta mostrar que existen barreras invisibles, pero efectivas, que permiten a las mujeres avanzar solo hasta un determinado nivel en la escala jerárquica de las organizaciones. Eagly y Carli (2007) sostienen que esta situación ha empezado a cambiar y que las mujeres presentan mayor acceso. Piénsese, por ejemplo, en los cargos políticos presidenciales ocupados por mujeres en los últimos tiempos. Estas autoras proponen el concepto de laberinto como nueva metáfora en reemplazo de la ya mencionada, destacando que, en la actualidad, a las mujeres no les está 
bloqueado el acceso a los puestos más altos, pero sí deben sortear diferentes obstáculos y recorrer dificultosos caminos para poder llegar a ellos.

Tradicionalmente se ha asociado al liderazgo con el género masculino. Los cargos directivos suelen ser caracterizados con propiedades generalmente atribuidas a los hombres como competitividad, control, autoridad u orientación hacia la tarea - rasgos instrumentales/ agénticos-. Estas consideraciones hacen que características propiamente femeninas como la orientación y preocupación por los otros - rasgos expresivos/ comunales- no sean consideradas esperables para los puestos de liderazgo, fomentando sesgos contra las mujeres en selección, promoción y ocupación de puestos directivos (Cheung \& Halpern, 2010; Cuadrado, 2004; Eagly 1987).

En esta línea, la Teoría de congruencia del rol hacia las mujeres líderes, propuesta por Eagly y Karau (2002), defiende que el prejuicio hacia las mujeres líderes es una de las causas de las dificultades que presentan las mujeres para acceder a puestos de liderazgo. El prejuicio procedería de la incongruencia entre las características femeninas y los requisitos del rol de líder. El constructo clave de la Teoría es el de roles de género, es decir, aquellas creencias compartidas acerca de los atributos de hombres y mujeres (Morales \& Cuadrado, 2004). Los roles incluyen dos tipos de expectativas o normas: descriptivas -expectativas compartidas sobre lo que los miembros de un grupo realmente hacen- y prescriptivas - expectativas compartidas sobre lo que los miembros de un grupo idealmente deberían hacer. En términos generales, las personas tienden a creer que para desempeñarse efectivamente en puestos de liderazgo es necesario desplegar cualidades masculinas, principalmente en roles definidos de modo más agéntico (e.g. roles militares). Dicha percepción ha presionado a muchas mujeres a adoptar características similares a los hombres. Sin embargo, cuando algunas mujeres llegan a ser consideradas igual de competentes que sus pares varones, las personas tienden a considerar que violan las normas esperadas socialmente (o prescriptivas) y reaccionan negativamente frente a ellas (Butler \& Geis, 1990). Por ende, las mujeres líderes pueden llegar a ser evaluadas negativamente por dos razones: ya sea porque no despliegan las características que suelen relacionarse con el liderazgo efectivo o porque, en el caso de que las desplieguen, son consideradas poco femeninas. De ambas formas existe incongruencia entre el rol de liderazgo y el rol social. En consecuencia se ven obligadas a combinar atributos asociados al estereotipo masculino (asertividad, competencia, control) con cualidades femeninas, con el fin de lograr no ser rechazadas principalmente por sus pares varones.

\section{TEORÍAS IMPLÍCITAS ACERCA DEL LIDERAZGO}

El estudio de las características esperadas para el correcto ejercicio del liderazgo es uno de los objetivos del Modelo denominado Procesamiento de la Información (Lord, Foti \& De Vader, 1984) que constituye un enfoque muy desarrollado en el abordaje de esta temática. Dicho modelo sostiene que, tanto líderes como subordinados, poseen un guión o estereotipo sobre cuáles son las conductas esperadas de una persona para ser considerada líder (Wofford, Godwin \& Wittington, 1998). Se sugiere que los miembros de un grupo de trabajo desarrollan, a través de experiencias pasadas con líderes, una serie de teorías implícitas acerca del liderazgo (TILs). Éstas consisten en un conjunto de presunciones acerca de atributos y habilidades que caracterizan a un líder ideal. Las TILs presuponen una serie de estructuras cognitivas o esquemas que especifican un repertorio de características que los seguidores esperan de sus líderes. Lord et al. (1984) argumentan que las diferentes percepciones que los seguidores sostienen acerca de sus superiores, conforman una serie de categorías cognitivas jerarquizadas, cada una de las cuales está representada por una serie de prototipos. Se encuentran condensadas en la memoria de los seguidores y se activan cuando interactúan con personas que ocupan una posición de liderazgo (Kenney, Schartz-Kenney \& Blascovich, 1996). Éstos esquemas proveen una estructura cognitiva que permite comprender los comportamientos de los superiores. Entonces, la esencia del liderazgo reside más en el seguidor que en 
las características individuales del propio líder. Así, un líder será considerado como tal si las características o conductas que de él perciben sus seguidores coinciden con los prototipos internalizados por ellos. Estos prototipos son altamente idiosincrásicos, por lo cual es esperable encontrar diferentes prototipos de líderes en diferentes grupos sociales y poblaciones.

En estudios previos sobre teorías implícitas acerca del liderazgo masculino y femenino (Lupano Perugini \& Castro Solano, 2008a,b) se identificaron Prototipos masculinos y femeninos de liderazgo sostenidos por líderes y seguidores. Se encontró, además, que a los prototipos masculinos se los relaciona con características como la capacidad de conducción y el logro de resultados obtenidos. En cambio, los prototipos femeninos eran asociados con atributos como la protección y la consecución de beneficios sociales, características que se corresponden con rasgos típicamente asociados al género femenino. Sin embargo, los participantes de dichos estudios consideraron que las líderes mujeres también despliegan características como la firmeza y la habilidad para superar obstáculos, las cuales son asociadas por lo general al género masculino. Dichos resultados dan cuenta de la necesidad de combinar atributos de ambos géneros para consolidar un liderazgo femenino efectivo. También se han hallado diferencias en los esquemas perceptuales de líderes y seguidores. Pudo observarse, que para ambos prototipos, los seguidores hacen mayor énfasis en aquellas características que se encuentran relacionadas con su posición, por ejemplo consideran que un líder efectivo debe tener en cuenta a los subalternos. En relación con las características de los prototipos femeninos fueron los seguidores quienes consideraron que las líderes mujeres deben combinar ambas características (comunales y agénticas), lo que da cuenta de procesos de categorización distintos en ambos tipos de participantes (líderes/ seguidores).

Como ya se mencionó, estos Prototipos son idiosincrásicos, investigaciones previas verificaron que la cultura organizacional influye sobre las TILs tanto de líderes y seguidores, resultando difícil la generalización a diferentes contextos organizacionales (Castro Solano, 2006; Castro Solano \& Lupano Perugini,
2005; Castro Solano \& Nader, 2004; House et. al, 1999). En relación con lo antedicho, se analizaron prototipos de liderazgo masculinos y femeninos en población militar, intentando dar cuenta de las características típicas que resultan deseables en ese contexto particular (Lupano Perugini, Castro Solano \& Casullo, 2008). En continuidad con dichas investigaciones, en este estudio, se intenta verificar si existen diferencias en las TILs de acuerdo al ámbito de desempeño del líder varón o mujer, en particular en los ámbitos empresariales, educacionales y políticos.

\section{LIDERAZGO, GÉNERO Y CONTEXTO}

Muchos autores sostienen que ciertas variables organizacionales tienen efecto en la manera de ejercer liderazgo. Estas variables son fundamentalmente la ocupación de un rol congruente con el género (trabajar en organizaciones típicamente femeninas para las mujeres y típicamente masculinas para los hombres) y el predominio numérico de hombres y mujeres en roles directivos (Eagly \& Jonson, 1990; Rosener, 1990; Gardiner \& Tiggerman, 1999, Van Engen, Van der Leeden \& Willemsen, 2001; Cuadrado, Navas \& Molero, $2004,2006)$. Cuadrado, Navas y Molero (2004) mostraron que las mujeres líderes consideran que desempeñan con mayor frecuencia un estilo de liderazgo democrático u orientado a las relaciones (considerado típicamente femenino, en contraposición a un estilo autoritario u orientado a la tarea considerado típicamente masculino) en organizaciones cuya actividad es típicamente femenina (e.g. organizaciones dedicadas a la salud o la educación, y empresas con rubros relacionados con la mujer, como ser la moda o la estética) que en las típicamente masculinas (e.g. entidades bancarias y empresas con rubros vinculados a los hombres, como la construcción o la producción automotriz). Cabe destacar que en ámbitos empresariales son pocas las mujeres que Ilegan a puestos directivos altos, en Argentina las CEO mujeres llegan apenas al $2 \%$ del total (Calvo, 2006; Casas 2010). Como ya ha sido demostrado por otras investigaciones (Druskat, 1994; Eagly \& Jonson, 1990; Rosener, 1990; Gardiner \& Tiggerman, 1999, Van Engen, Van der Leeden \& Willemsen, 2001), el tipo de actividad no es determinante por sí misma del 
estilo de liderazgo a ser ejercido sino en combinación con otras variables. Por ello, cuando se combinan los dos criterios de género (tipo de actividad y dominación numérica de hombres y mujeres en puestos directivos), se observa que la congruencia entre el sexo del líder (mujer), el tipo de organización (femenina) y el predominio numérico en puestos directivos (mujeres) produce una mayor utilización de estilos de liderazgo congruentes con el estereotipo femenino (e.g. democrático). Ridgeway (2001) sostiene, en relación con lo planteado, que los comportamientos de hombres y mujeres varían en virtud del tipo de cultura organizacional, del tipo de tareas a desarrollar y de la composición sexual del plantel de empleados.

Específicamente en relación al ámbito educacional, si bien hay un mayor número de mujeres en cargos directivos en comparación con otros ámbitos, resulta inferior al que correspondería en proporción a su presencia en esta profesión. Ocupan mayoritariamente puestos de dirección en nivel inicial y primario. Parece ser que a mayor nivel educativo, menor proporción de mujeres en puestos directivos. Además, parece existir mayor credibilidad por parte del profesorado y alumnado hacia los directivos hombres (Diez Gutierrez, Terrón Bañuelos, Valle Flórez \& Centeno Suárez, 2002).

En las organizaciones universitarias, en los últimos años, las mujeres han incrementado notablemente la matrícula y el plantel docente, pero no los cargos directivos (Glazer-Raymo, 1999; Ropers-Huilman, 2003). Sánchez Moreno y López Yáñez (2008) realizaron un trabajo acerca de mujeres que se desempeñan en cargos de gestión universitaria atinente a indagar datos acerca del perfil profesional de las mismas, roles desempeñados, estilos de liderazgo, problemas y necesidades formativas percibidas por ellas. Dentro de los resultados obtenidos se destaca la percepción, por parte de las líderes, de la adopción de un estilo de liderazgo flexible, que se adapta al contexto y a la situación, y fuertemente orientado al grupo y su bienestar, que combina tanto aspectos sociales como técnicos del ejercicio de los cargos de gestión. Al ser consultadas por las características que consideran deben ser valoradas para el correcto desempeño del puesto, privilegiaron la responsabilidad, la capacidad de planificación y organización, capacidad de resolución de conflictos y sentido ético. Los seguidores también señalaron la responsabilidad como la característica más valorada, pero destacaron que percibían en sus directivas poca capacidad de gestión económica.

Por último, en relación al ámbito político, cada vez son más las mujeres que presentan sus candidaturas. Distintos estudios muestran que las mujeres que ocupan cargos políticos gozan de una creciente aceptación por parte del electorado (Carroll, 1985; Darcy, Welch \& Clark, 1987). Benze y Declerq (1985) sintetizaron las características que suelen preferir los electores: mujeres fuertes pero no agresivas, que muestran perfil profesional y que no son demasiado atractivas físicamente. No existe acuerdo acerca de si difieren de los hombres respecto de su estilo de liderar. Genovese (1993) estudió varias líderes políticas y señaló que no existen diferentes estilos, sino situaciones diferentes que requieren de la adaptación de los propios estilos. Uriarte y Ruiz (1999) sostienen que las políticas son más dialogantes, menos autoritarias y tienen mayor capacidad de integrar a los subordinados. Benze y Declerq (1985) realizaron un análisis de contenido de spots electorales y concluyeron que en ellos los hombres tienden a enfatizar la dureza, mientras que las mujeres usualmente acentúan su capacidad de compasión.

En Argentina, D'Adamo, García Beaudoux, Ferrari y Slavinsky (2008) realizaron un estudio tendiente a explorar las percepciones que predominan en la opinión pública acerca del liderazgo femenino, específicamente, de las mujeres que presentan candidaturas políticas. Los resultados mostraron que la mayoría considera que no hay diferencias en cuanto a estilos de liderazgo entre hombres y mujeres, que ellas se preocupan más por las cuestiones sociales y que presentan mayores dificultades y son más criticadas ya que existen prejuicios negativos hacia ellas. Por último consideran que existen atributos que favorecen a las mujeres en campaña: demostrar inteligencia (para negociar), paciencia, firmeza, decisión y saber explotar sus cualidades físicas y femeninas en cuanto a capacidad de empatía y comprensión.

En función de lo previamente mencionado, el presente estudio tiene los siguientes objetivos: 
1. Verificar si existen diferencias significativas en las teorías implícitas acerca del liderazgo masculino y femenino según ámbito de desempeño (empresarial, académico y político), en población argentina.

2. Analizar, diferencias según ámbito de desempeño (empresarial, académico y político) $y$, además, según sexo de quien responde, para el caso de las teorías implícitas acerca del liderazgo femenino, en población argentina.

\section{MÉTODO}

\subsection{Participantes}

Participaron de forma voluntaria 441 sujetos adultos, 222 varones $(50,3 \%)$ y 219 mujeres $(49,7 \%)$ que tenían en promedio 32,38 años (DE $=10,14)$. Si bien la selección no se realizó al azar, se convocó a gran cantidad de pasantes de la Universidad sede del trabajo para que recolectaran los datos de la manera más aleatoria más posible.

En relación a la ocupación laboral se definieron cinco grupos. El 16,1\% eran funcionarios, directivos y/o profesionales -se trataba de la categoría ocupacional más alta- $(n=71)$. El $10,7 \%$ eran jefes de nivel intermedio $(n=$ $46)$. El $54,9 \%(n=242)$ trabajaba como comerciantes, empleados calificados, docentes, entre otros. El 16,6\% ( $n=73)$ trabajaba como cuentapropistas, trabajadores de servicios, choferes, empleados no calificados, entre otros. El $2 \%$ restante se dedicaba a actividades no incluidas en las categorías precedentes $(\mathrm{n}=$ 9). Los participantes refirieron que se desempeñaban principalmente en grandes empresas o multinacionales $(41 \%, \mathrm{n}=181)$, en medianas empresas el $31,3 \%(n=138)$ y en pequeñas empresas el $25,2 \%(n=111)$. El 2,5\% $(n=11)$ no refirió datos.

Con respecto al nivel educativo, la mayoría $(70,2 \%)$ estaba cursando o poseía estudios universitarios o superiores $(n=308)$. El 13,6\% $(n=60)$ refería estar cursando o haber finalizado estudios terciarios; y el $16,6 \%$ restante presentaba un nivel de estudios menor -principalmente secundario completo- $(n=73)$.

La mayor parte de los participantes (85,4\%; $\mathrm{n}=377$ ) residían en la ciudad de Buenos Aires y el conurbano bonaerense (Argentina). El resto $(11,6 \%)$ se distribuía en provincias del interior, y solo una persona residía temporalmente en el exterior. Se decidió dejar a la persona extranjera ya que reside de forma alternada en Argentina y en el exterior y esta particularidad es cada vez mas frecuente en las grandes urbes como Buenos Aires. Lo mismo sucedía con las personas que tenían lugar de residencia en el interior del país. El 2,7\% ( $n=$ 12) no refirió lugar de residencia.

\subsection{Instrumentos}

Listado de Atributos de liderazgo según ámbito de desempeño (Formas Mujer- Varón)

En un estudio anterior (Lupano Perugini \& Castro Solano, 2008a) se identificaron Prototipos masculinos y femeninos de liderazgo efectivo a partir de la administración de un protocolo cualitativo diseñado ad- hoc que constaba de los siguientes elementos:

1. Nombre del líder. Se solicitaba a los participantes que nombren a una persona percibida por ellos como líder efectivo. En primer lugar se les pedía que nombren a un líder varón y, luego, a una líder mujer;

2. Justificación. En una pregunta abierta se pedía a las personas que describieran con sus palabras, las razones o características por las cuales consideraban como líder al sujeto (varón y mujer) nombrado anteriormente.

En dicho estudio se generó un listado de categorías de tipos de líder (e.g. líder político, militar, religioso, etc) y nombres (e.g. Perón, Madre Teresa de Calcuta) que constituyen ejemplares de líderes efectivos hombres y mujeres. En esa misma investigación se analizaron las características que abarcaban dichos prototipos. Para realizar dicho análisis se trabajó sobre la segunda parte del instrumento de recolección de datos (Solicitud de justificación de la elección realizada en la primera parte). Se realizó un análisis de contenido de las respuestas dadas para líderes varones y mujeres, generando veinticinco categorías (exclusivas y excluyentes) de características, de acuerdo a los atributos que eran mencionados con mayor frecuencia. 
Posteriormente se procedió a recategorizar las respuestas asignando la categoría que mayormente representara a la respuesta dada por el sujeto. Las categorías generadas, se presentan en la Tabla 1.

Para la presente investigación se tomaron 23 de las características listadas (se eliminó "Logros similares a los hombres" ya que es una categoría exclusiva del liderazgo femenino, y "Hazañas y hechos históricos" porque resultaba no aplicable a los propósitos de indagación de este estudio).

Se diseñaron seis protocolos. Tres de ellos solicitaban al participante que pensara en una mujer que ocupara un puesto jerárquico y que marcara en una escala de 1 a 7 el grado en el que cree que debe predominar cada una de las 23 características listadas para ser considerada una líder efectiva. Se le pedía que esa elección la hiciera pensando en tres ámbitos de desempeño: un protocolo indagaba sobre el desempeño en el ámbito empresarial, otro en el ámbito político y el último en el ámbito académico. Los otros tres protocolos presentaban la misma consigna pero se le pedía al participante que pensara en un líder hombre. Para la recolección de datos se pidió a la mitad de los participantes que contestaran a los tres protocolos que indagaban sobre la líder mujer (Forma Mujer) y, a la otra mitad, sobre el líder hombre (Forma Hombre).

\section{PROCEDIMIENTO}

Los instrumentos formaban parte de una batería de pruebas más amplia que se administró con el propósito de obtener información acerca de teorías implícitas, creencias y actitudes acerca del liderazgo femenino y masculino. Las otras pruebas administradas fueron: CEALM -Escala de creencias estereotipadas acerca de mujeres líderes - (Moore, Grunberg \& Greenberg, 2003); ACT- ML - Escala de actitudes hacia las mujeres líderes - (Lupano Perugini \& Castro Solano, 2010).

Las tareas de supervisión de recolección y entrada de datos estuvieron a cargo de un becario. El análisis de los datos se efectuó con el procesador SPSS versión 13.0.

\section{RESULTADOS}

Analizando los objetivos planteados, los resultados en cada caso son:

Diferencias en las teorías implícitas acerca del liderazgo masculino y femenino según ámbito de desempeño (empresarial, académico y político).

A fin de responder al primer objetivo que se propone verificar la existencia de diferencias estadísticamente significativas en las teorías implícitas acerca del liderazgo masculino y femenino según ámbito de desempeño (empresarial, académico y político), se llevó a cabo un análisis multivariado de la varianza (MANOVA) con un diseño 2 (Forma TILs = Forma Mujer o Forma Hombre) $\times 3$ (Ámbito = empresarial, académico y político).

En cuanto a la influencia del Ámbito, el análisis realizado arrojó diferencias estadísticamente significativas entre los vectores de medias de las TILs para los diferentes contextos $(\lambda=0.40, F$ $(46,2590)=32.39, p<0.001, \eta=0.36)$. Por lo tanto el contraste de las medias arroja diferencias en la mayoría de las TILs. Siguiendo las indicaciones de Cohen (1991) para la interpretación del tamaño del efecto del contraste multivariado implementado podemos considerar la magnitud de estas diferencias como amplia.

Tabla 1.

Recategorización de las respuestas

\begin{tabular}{ll}
\hline Capacidad de adaptación & Ejemplo personal \\
Capacidad de conducción & Entrega, sacrificio y compromiso \\
Capacidad de enseñanza & Firmeza y superación de obstáculos \\
Capacidad de motivar & Hazañas y hechos históricos \\
Capacidad de resolver problemas & Honestidad y valores \\
Capacidad estratégica y de negociación & Inteligencia \\
Carisma y ascendiente & Logros similares a los hombres (sólo para los \\
& prototipos femeninos de liderazgo) \\
Claridad en los objetivos & Prestigio y reconocimiento \\
Consecución de beneficios sociales & Protección \\
Consideración de los demás & Responsabilidad \\
Constancia y perseverancia & Resultados obtenidos \\
Creatividad e innovación & Visionario \\
Dominancia y poder & \\
\hline
\end{tabular}


El análisis no arrojó diferencias estadísticamente significativas entre los vectores de medias de las TILs teniendo en cuenta la Forma $(\lambda=$ $0.97, F(23,1295)=1.25, p>0.05, \eta=0.02)$. Por lo tanto puede afirmarse que las TILs se mantienen estables y no se ven afectadas por la variable Forma, es decir que no difieren según refieran al liderazgo masculino o femenino.

No se observó interacción entre el Ámbito y la Forma $(\lambda=0.97, F(46,2590)=0.64, p>0.05$, $\eta=0.01$ ). Por lo tanto no existen diferencias en las TILs considerando la Forma (TILs acerca del liderazgo masculino o femenino) y el Ámbito (empresarial, académico y político) tomados en forma conjunta.

A fin de examinar las diferencias individuales en las TILs según contexto se realizaron contrastes univariados registrándose diferencias en la mayoría de ellas tal como se muestra en la Tabla 2.

Puede observarse en la Tabla 2 que determi- nadas características resultan más relevantes para los ámbitos empresariales y políticos que para los académicos, como ser: capacidad de adaptación, de conducción, de resolver problemas, estratégica y de negociación, firmeza y superación de obstáculos y ser visionario. En cambio, en el académico, se destacan las cualidades de capacidad de enseñanza, inteligencia y de entrega, sacrificio y compromiso (comparado especialmente con el ámbito empresarial). En la empresa parece ser menos influyente el prestigio y reconocimiento (comparando con los otros ámbitos). En la política resulta relevante el carisma y ascendiente, la protección, la consideración de los demás, la consecución de beneficios sociales, y la dominancia y poder. En cambio parece ser menos importante la capacidad de motivar y la creatividad e innovación (comparando con los otros ámbitos).

Diferencias según ámbito de desempeño (empresarial, académico y político) y, sexo de quien responde, para el caso de las teorías implícitas acerca del liderazgo femenino.

Tabla 2.

Diferencias individuales en las TILs según sexo.

\begin{tabular}{|c|c|c|c|c|c|}
\hline \multirow[t]{2}{*}{ Atributo } & \multicolumn{3}{|c|}{ Media (DE) } & \multirow{2}{*}{$\begin{array}{c}\text { ANOVA } \\
F\end{array}$} & \multirow[t]{2}{*}{ Tukey-b } \\
\hline & $\begin{array}{l}\text { Empresarial } \\
\text {-Grupo a- }\end{array}$ & $\begin{array}{l}\text { Politico } \\
\text {-Grupo b- }\end{array}$ & $\begin{array}{l}\text { Académico } \\
\text {-Grupo c- }\end{array}$ & & \\
\hline Capacidad de adaptación & $5,91(1,16)$ & $5,88(1,31)$ & $5,58(1,13)$ & $9,29 * *$ & Grupos a-b y c \\
\hline Capacidad de conducción & $6,47(0,88)$ & $6,46(0,99)$ & $5,99(1,2)$ & $31,49^{* *}$ & Grupos a-b y c \\
\hline Capacidad de enseñanza & $5,62(1,3)$ & $4,79(1,6)$ & $6,66(0,87)$ & $237,99^{* *}$ & Grupos a y by c \\
\hline Capacidad de motivar & $6,26(1,1)$ & $5,89(1,31)$ & $6,35(1)$ & $20,02^{* *}$ & Grupos a-c y b \\
\hline Capacidad de resolver problemas & $6,41(0,98)$ & $6,26(1,19)$ & $5,85(1,16)$ & $30,68^{* *}$ & Grupos a-b y c \\
\hline $\begin{array}{l}\text { Capacidad estratégica y de } \\
\text { negociación }\end{array}$ & $6,34(0,94)$ & $6,49(0,93)$ & $4,83(1,52)$ & $277,02^{* *}$ & Grupos a-b y c \\
\hline Carisma y ascendiente & $5,72(1,21)$ & $6,25(1,17)$ & $5,47(1,39)$ & $44,57^{* *}$ & Grupos a y b y c \\
\hline Claridad en los objetivos & $6,39(0,87)$ & $6,28(1,16)$ & $6,21(1,07)$ & $3,45^{*}$ & Grupos a y c \\
\hline Consecución de beneficios sociales & $5,11(1,4)$ & $6,09(1,24)$ & $4,93(1,57)$ & $87,08^{* *}$ & Grupos b y c \\
\hline Consideración de los demás & $5,48(1,3)$ & $5,84(1,46)$ & $5,65(1,31)$ & $7,66^{* *}$ & Grupos a y b \\
\hline Constancia y perseverancia & $5,92(1,08)$ & $5,87(1,23)$ & $5,99(1,13)$ & $1,16 n s$ & - \\
\hline Creatividad e innovación & $5,88(1,15)$ & $5,42(1,36)$ & $6,06(1,19)$ & $30,77^{* *}$ & Grupos a-c y b \\
\hline Dominancia y poder & $5,37(1,5)$ & $5,9(1,36)$ & $4,46(1,64)$ & $102,85^{* *}$ & Grupos a y b y c \\
\hline Ejemplo personal & $5,79(1,34)$ & $5,77(1,5)$ & $5,8(1,4)$ & $0,04 n s$ & - \\
\hline Entrega, sacrificio y compromiso & $5,8(1,24)$ & $5,95(1,39)$ & $6,04(1,18)$ & $3,82^{*}$ & Grupos a y c \\
\hline Firmeza y superación de obstáculos & $6,17(0,97)$ & $6,1(1,17)$ & $5,76(1,16)$ & $17,42^{* *}$ & Grupos a-b y c \\
\hline Honestidad y valores & $6,09(1,21)$ & $6,17(1,35)$ & $5,99(1,27)$ & $2,1 n s$ & - \\
\hline Inteligencia & $6,15(0,97)$ & $6,16(1,05)$ & $6,44(0,87)$ & $12,68^{* *}$ & Grupos a-b y c \\
\hline Prestigio y reconocimiento & $5,51(1,23)$ & $5,85(1,22)$ & $5,86(1,23)$ & $11,65^{* *}$ & Grupos b-c y a \\
\hline Protección & $4,97(1,44)$ & $5,37(1,38)$ & $4,97(1,5)$ & $10,94^{* *}$ & Grupos a-c y b \\
\hline Responsabilidad & $6,44(0,87)$ & $6,34(1,05)$ & $6,44(0,87)$ & $1,72 n s$ & - \\
\hline Obtención de buenos resultados & $6,16(1,99)$ & $6,18(1,15)$ & $6,13(1,05)$ & $0,24 n s$ & - \\
\hline Visionario & $5,88(1,21)$ & $6,06(1,23)$ & $5,44(1,47)$ & $26,17^{* *}$ & Grupos a-b y c \\
\hline
\end{tabular}


A fin de responder al segundo objetivo que se propone verificar la existencia de diferencias según ámbito de desempeño (empresarial, académico y político) y, además, según sexo de quien responde, para el caso de las teorías implícitas acerca del liderazgo femenino, se llevó a cabo un análisis multivariado de la varianza (MANOVA) con un diseño 2 (Sexo = mujer o varón) $x$ 3 (Ámbito = empresarial, académico y político).

En cuanto a la influencia del Ámbito, el análisis realizado arrojó diferencias estadísticamente significativas entre los vectores de medias de las TILs para los diferentes contextos $(\lambda=0.39$, $F(46,1264)=16,54, p<0.001, \eta=0.37)$. Por lo tanto el contraste de las medias arroja diferencias en la mayoría de las TILs. Siguiendo las indicaciones de Cohen (1991) para la interpretación del tamaño del efecto del contraste multivariado implementado podemos considerar la magnitud de estas diferencias como amplia.

El análisis, también arrojó diferencias estadísticamente significativas entre los vectores de medias de las TILs teniendo en cuenta el sexo de quien responde $(\lambda=0.93, F(23,632)=1.98$, $p>0.05, \eta=0.06)$. Por lo tanto puede afirmarse que las TILs difieren según el perceptor sea una mujer o un hombre, aunque las diferencias son consideradas pequeñas.

No se observó interacción entre el Ámbito $y$ el Sexo $(\lambda=0.92, F(46,1264)=1.02, p>$ $0.05, \eta=0.03)$. Por lo tanto no existen diferencias en las TILs considerando el Ámbito (empresarial, académico y político) y el sexo del perceptor, tomados en forma conjunta.

A fin de examinar las diferencias individuales en las TILs acerca del liderazgo femenino según contexto se realizaron contrastes univariados registrándose diferencias en la mayoría de ellas tal como se muestra en la Tabla 3.

En virtud de que los análisis realizados para el cumplimiento del Objetivo 1 arrojaron que no existen diferencias en las TILs según la Forma (Forma Mujer o Forma Hombre), las diferencias

Tabla 3.

Diferencias individuales en las TILs acerca del liderazgo femenino según contexto.

\begin{tabular}{|c|c|c|c|c|c|}
\hline \multirow[t]{2}{*}{ Atributo } & \multicolumn{3}{|c|}{ Media (DE) } & \multirow{2}{*}{$\begin{array}{l}\text { ANOVA } \\
\quad F\end{array}$} & \multirow[t]{2}{*}{ Tukey-b } \\
\hline & $\begin{array}{l}\text { Empresarial } \\
\text {-Grupo a- }\end{array}$ & $\begin{array}{l}\text { Político } \\
\text {-Grupo b- }\end{array}$ & $\begin{array}{l}\text { Académico } \\
\text {-Grupo c- }\end{array}$ & & \\
\hline Capacidad de adaptación & $5,90(1,15)$ & $5,78(1,39)$ & $5,53(1,35)$ & $4,71^{\star *}$ & Grupos a y c \\
\hline Capacidad de conducción & $6,42(0,95)$ & $6,36(1,08)$ & $5,96(1,23)$ & $11,4^{* *}$ & Grupos a-b y c \\
\hline Capacidad de enseñanza & $5,64(1,23)$ & $4,82(1,56)$ & $6,63(0,92)$ & $113,47^{* *}$ & Grupos a y b y $c$ \\
\hline Capacidad de motivar & $6,20(1,17)$ & $5,86(1,13)$ & $6,35(1,08)$ & $9,91^{\star *}$ & Grupos $a-c$ y b \\
\hline Capacidad de resolver problemas & $6,40(1,03)$ & $6,23(1,23)$ & $5,81(1,19)$ & $14,95^{* *}$ & Grupos a-b y c \\
\hline $\begin{array}{l}\text { Capacidad estratégica y de } \\
\text { negociación }\end{array}$ & $6,25(1,03)$ & $6,39(1)$ & $4,83(1,53)$ & $110,54^{* *}$ & Grupos a-b y c \\
\hline Carisma y ascendiente & $5,73(1,19)$ & $6,20(1,14)$ & $5,38(1,36)$ & $24,47^{* *}$ & Grupos a y b y c \\
\hline Claridad en los objetivos & $6,36(0,95)$ & $6,21(1,21)$ & $6,16(1,08)$ & $1,91 n s$ & - \\
\hline Consecución de beneficios sociales & $5,01(1,47)$ & $6,06(1,23)$ & $4,90(1,5)$ & $45,21^{* *}$ & Grupos $a-c$ y b \\
\hline Consideración de los demás & $5,5(1,21)$ & $5,85(1,4)$ & $5,7(1,24)$ & $4,07^{*}$ & Grupos a y b \\
\hline Constancia y perseverancia & $5,95(1,06)$ & $5,85(1,22)$ & $5,98(1,2)$ & $0,8 n s$ & - \\
\hline Creatividad e innovación & $5,95(1,11)$ & $5,45(1,32)$ & $6,05(1,21)$ & $14,8^{* *}$ & Grupos a-c y b \\
\hline Dominancia y poder & $5,37(1,55)$ & $5,91(1,38)$ & $4,48(1,59)$ & $49,8^{* *}$ & Grupos a y b y c \\
\hline Ejemplo personal & $5,84(1,29)$ & $5,7(1,48)$ & $5,8(1,38)$ & $0,57 n s$ & - \\
\hline Entrega, sacrificio y compromiso & $5,83(1,21)$ & $5,91(1,42)$ & $6,03(1,2)$ & $1,29 n s$ & - \\
\hline Firmeza y superación de obstáculos & $6,19(1,02)$ & $6,1(1,21)$ & $5,75(1,2)$ & $8,94^{* *}$ & Grupos a-b y c \\
\hline Honestidad y valores & $6,15(1,13)$ & $6,11(1,41)$ & $5,96(1,35)$ & $1,32 n s$ & - \\
\hline Inteligencia & $6,15(0,97)$ & $6,25(0,94)$ & $6,42(0,87)$ & $4,82^{* *}$ & Grupos a y c \\
\hline Prestigio y reconocimiento & $5,48(1,16)$ & $5,85(1,22)$ & $5,8(1,3)$ & $5,64^{* *}$ & Grupos b-c y a \\
\hline Protección & $4,92(1,42)$ & $5,36(1,35)$ & $5(1,14)$ & $6,24^{* *}$ & Grupos a-c y b \\
\hline Responsabilidad & $6,43(0,96)$ & $6,4(0,97)$ & $6,51(0,8)$ & $0,89 n s$ & - \\
\hline Obtención de buenos resultados & $6,11(1,05)$ & $6,2(1,16)$ & $6,11(1,06)$ & $0,45 n s$ & - \\
\hline Visionaria & $5,85(1,19)$ & $6,04(1,26)$ & $5,41(1,45)$ & $13,06^{\star *}$ & Grupos a-b y c \\
\hline
\end{tabular}


halladas en las TILs acerca del liderazgo femenino son muy similares a las halladas tomando las TILs en conjunto (Forma Mujer y Forma Hombre). Por lo tanto, se halló que determinadas características resultan más relevantes para los ámbitos empresariales y políticos que para los académicos, como por ejemplo: capacidad de adaptación, de conducción, de resolver problemas, estratégica y de negociación, firmeza y superación de obstáculos y ser visionaria. En el académico, en cambio, también se destacaron las cualidades de capacidad de enseñanza e inteligencia. En la empresa también resultó ser menos influyente el prestigio y reconocimiento (comparando con los otros ámbitos). Por último, en la política también resultó relevante el carisma y ascendiente, la consideración de los demás, protección, la consecución de beneficios sociales, y la dominancia y poder. En cambio pareció ser menos importante la capacidad de motivar y la creatividad e innovación (comparando con los otros ámbitos).

Por último, con la finalidad de examinar las diferencias individuales en las TILs acerca del liderazgo femenino según sexo, se realizaron contrastes univariados registrándose diferencias en la mayoría de ellas tal como se muestra en la Tabla 4.

Los resultados exhibidos en la Tabla 4 muestran que todas las diferencias estadísticamente significativas fueron halladas a favor de las mujeres. Es decir que las participantes de esta investigación consideran que las mujeres líderes muestran, en mayor medida que los hombres, casi todos los atributos listados. Llama la atención que no se hallaron diferencias en aquellos atributos que son típicamente femeninos como por ejemplo la protección, la entrega, sacrificio y compromiso.

\section{DISCUSIÓN}

Este trabajo presentaba el interés de señalar la importancia del contexto en el ejercicio del liderazgo. Quedó demostrado que el ámbito de desempeño determina diferencias significativas

Tabla 4.

Diferencias individuales en las TILs acerca del liderazgo femenino según sexo.

\begin{tabular}{|c|c|c|c|}
\hline \multirow[t]{2}{*}{ Atributo } & \multicolumn{2}{|c|}{ Media (DE) } & \multirow{2}{*}{$\begin{array}{c}\text { ANOVA } \\
F\end{array}$} \\
\hline & Varón & Mujer & \\
\hline Capacidad de adaptación & $5,65(1,25)$ & $5,81(1,35)$ & $2,28 n s$ \\
\hline Capacidad de conducción & $6,12(1,15)$ & $6,37(1,07)$ & $8,74^{* *}$ \\
\hline Capacidad de enseñanza & $5,63(1,47)$ & $5,75(1,47)$ & $1,07 n s$ \\
\hline Capacidad de motivar & $5,98(1,27)$ & $6,28(1,15)$ & $9,85^{* \star}$ \\
\hline Capacidad de resolver problemas & $6,02(1,27)$ & $6,25(1,09)$ & $6,3^{*}$ \\
\hline $\begin{array}{l}\text { Capacidad estratégica y de } \\
\text { negociación }\end{array}$ & $5,64(1,45)$ & $5,98(1,34)$ & $9,36^{*}$ \\
\hline Carisma y ascendiente & $5,63(1,3)$ & $5,91(1,25)$ & $7,95^{* *}$ \\
\hline Claridad en los objetivos & $6,09(1,17)$ & $6,38(1)$ & $11,72^{\star *}$ \\
\hline Consecución de beneficios sociales & $5,14(1,55)$ & $5,49(1,44)$ & $9,24^{* *}$ \\
\hline Consideración de los demás & $5,51(1,33)$ & $5,84(1,24)$ & $11^{\star \star}$ \\
\hline Constancia y perseverancia & $5,72(1,21)$ & $6,1(1,09)$ & $17,87^{* *}$ \\
\hline Creatividad e innovación & $5,7(1,25)$ & $5,92(1,24)$ & $5^{*}$ \\
\hline Dominancia y poder & $5,12(1,62)$ & $5,38(1,61)$ & $4,17^{*}$ \\
\hline Ejemplo personal & $5,63(1,42)$ & $5,91(1,35)$ & $7^{* *}$ \\
\hline Entrega, sacrificio y compromiso & $5,83(1,28)$ & $6,01(1,28)$ & $3,09 n s$ \\
\hline Firmeza y superación de obstáculos & $5,85(1,21)$ & $6,16(1,1)$ & $11,75^{\star \star}$ \\
\hline Honestidad y valores & $5,9(1,4)$ & $6,23(1,2)$ & $10,18^{* \star}$ \\
\hline Inteligencia & $6,14(0,99)$ & $6,39(0,87)$ & $12,15^{\star *}$ \\
\hline Prestigio y reconocimiento & $5,58(1,21)$ & $5,82(1,25)$ & $5,99^{*}$ \\
\hline Protección & $5,06(1,35)$ & $5,13(1,46)$ & $0,35 n s$ \\
\hline Responsabilidad & $6,27(1,02)$ & $6,6(0,77)$ & $22,99^{\star \star x}$ \\
\hline Obtención de buenos resultados & $6,02(1,11)$ & $6,24(1,07)$ & $6,97^{* *}$ \\
\hline Visionaria & $5,62(1,34)$ & $5,9(1,32)$ & $7,22^{* *}$ \\
\hline
\end{tabular}


en las teorías implícitas acerca del liderazgo. Sin embargo, los resultados mostraron que no existen diferencias según el ámbito de desempeño en cuanto a Prototipos masculinos o femeninos de liderazgo.

Por lo tanto, en relación con los resultados previos obtenidos (Lupano Perugini \& Castro Solano, 2008a), puede decirse que existen diferencias entre Prototipos de liderazgo masculinos y femeninos pero, que si se los analiza más específicamente según ámbito de desempeño (empresarial, académico y político), no se hallan discrepancias. Es decir que puede afirmarse que el contexto y la cultura organizacional influyen sobre las TILs de líderes y seguidores (Castro Solano, 2006; House et. al, 1999), pero a nivel general, sin tener en cuenta el sexo del líder.

Es probable que los líderes pongan en práctica diferentes conductas y estrategias en función del contexto en el que les toca desempeñarse, por lo tanto un estilo de liderazgo puede ser más adaptativo en un contexto que en otro (Castro Solano \& Nader, 2004). Además, puede afirmarse que la cultura organizacional en donde están insertos tanto seguidores como líderes modera e influye sobre la percepción de aquel que tiene que caracterizar a un líder efectivo (House, Javidan \& Dorfman, 2001).

En el caso particular de los resultados hallados en la presente investigación, se encontró que las características que son asignadas a líderes efectivos, varían según el lugar dónde este se desempeñe. Para el caso del ámbito académico resultaron poco relevantes atributos relacionados con la capacidad estratégica de negociación, de resolver problemas o tener visión de futuro. Estas características resultan mayormente deseables para los ámbitos políticos y empresariales. En el entorno educativo, las cualidades que son elegidas para describir a un líder eficaz son las relacionadas con el conocimiento, como ser la inteligencia y, también, tener la capacidad de transmitir o enseñar. En la política se destacan todas aquellas características que están relacionadas con el bienestar general de la población, como ser la consecución de beneficios sociales y la consideración de los demás. También en este ámbito resulta muy importante la capacidad del líder de poder influir sobre otros, por ello de destacan el carisma, la dominancia y el poder del líder. Seguramente estos atributos sean valorados en virtud de que en este ámbito el líder debe lograr tener injerencia sobre grandes grupos, siendo menos importante, por ejemplo, la creatividad del dirigente, atributo mucho más valorado en la educación.

El presente trabajo intentaba también verificar diferencias según ámbito de desempeño y según sexo de quien responde, para el caso de las teorías implícitas acerca del liderazgo femenino. Las diferencias halladas para las TILs acerca del liderazgo femenino resultaron ser casi las mismas que para las TILs tomadas en conjunto, ya que no fueron verificadas diferencias según forma (líder varón o mujer), tal como se expuso previamente.

Se puede ver que el atributo responsabilidad que resultó ser ampliamente valorado en investigaciones previas relacionadas al ámbito académico (Sánchez Moreno \& López Yáñez, 2008), en el presente trabajo no fue una característica que generara diferencias entre los ámbitos, sino que parece ser significativa para todos ellos. En esta investigación, se destacaron los factores vinculados al conocimiento y a su transmisión y no tanto a las habilidades de gestión (e.g. capacidad de planificación y organización) como en la investigación citada. En cambio, en la política y la empresa, esas capacidades resultaron más deseadas. Para los participantes de esta investigación, hombres y mujeres no diferirían en sus atributos a la hora de liderar -tal como sostiene Genovese, 1993-, sino que ambos requerirían de cualidades de ascendencia y preocupación por las causas sociales.

Resultó relevante en este estudio las diferencias halladas según sexo de quien responde dando cuenta de procesos de categorización distintos entre hombres y mujeres. Estas últimas, al ser consultadas por las características de Prototipos de líderes mujeres, asumieron que las líderes presentan, en mayor medida, casi todas las características presentadas. Llamó la atención que no se hallaran diferencias en aquellos atributos que son típicamente femeninos como por ser la protección, la entrega, sacrificio y compromiso. Estos resultados indican, como sostienen Cuadrado, Navas y Molero (2004), que con independencia del sexo del líder y del tipo de organización, el sexo del evaluador también puede tener importancia en ciertas ocasiones. 
Puede considerarse entonces que, si no existen diferencias en las TILs acerca del liderazgo masculino y femenino según ámbito de desempeño, las diferencias entre hombres y mujeres en el acceso a los puestos directivos siga siendo resultado de la existencia de prejuicios. D'Adamo, García Beaudoux, Ferrari y Slavinsky (2008), en su investigación sobre liderazgo femenino en política, encontraron que casi el $60 \%$ de los participantes consideraron que entre los votantes existen prejuicios negativos hacia las candidatas mujeres. Muchos contextos organizacionales, especialmente los empresariales, pueden promover, fomentar o permitir determinados estilos de liderazgo o características que pueden perjudicar a las mujeres líderes (Cuadrado, Navas \& Molero, 2004). Es decir, determinados ámbitos privilegian el poseer cualidades típicamente masculinas (como la capacidad estratégica o la dominancia, previamente mencionadas) lo cual favorece el acceso de los hombres a los puestos más altos por no presentar incongruencia entre su rol de líder y su rol social, tal como propone la Teoría de la congruencia de rol previamente descripta (Eagly \& Karau, 2002).

Futuras investigaciones deberán dar cuenta de si existen diferencias en TILs acerca del liderazgo femenino según el tipo de actividad desarrollada, principalmente en las empresas, ya que trabajos previos (e.g. Van Engen, Van der Leeden \& Willemsen, 2001; Cuadrado, Navas \& Molero, 2004) mostraron que las mujeres suelen desarrollar un estilo de liderazgo más congruente con el estereotipo femenino cuando se desempeñan en empresas con rubros típicamente femeninos (e.g. empresas de belleza o dedicadas a la educación).

También deberían profundizarse las investigaciones acerca del liderazgo femenino en política ya que se evidencia que las características que suelen ser percibidas en ese ámbito son una combinatoria de atributos típicamente masculinos (e.g. dominancia) y femeninos (e.g. protección), lo cual resulta congruente con los Prototipos femeninos de liderazgo efectivo (e.g. Eagly \& Karau, 2002; Lupano Perugini \& Castro Solano, 2008a). Esto quizás explique la creciente aceptación, por parte del electorado de candidatas mujeres (Carroll, 1985; Darcy, Welch \& Clark, 1987; D’Adamo, García Beaudoux, Ferrari \& Slavinsky, 2008; Eagly \& Carli, 2007). Algunos cambios actuales en las organizaciones, como por ejemplo la preferencia de estilos más democráticos de liderazgo pueden favorecer a las mujeres (Eagly \& Karau, 2002), aunque debe tenerse en cuenta que muchas veces las cualidades típicamente femeninas o comunales (como la orientación hacia otros) pueden ser vistas como ventajas y desventajas simultáneamente. D’Adamo, García Beaudoux, Ferrari y Slavinsky (2008), hallaron que, por un lado se destaca en las mujeres candidatas sus habilidades interpersonales y de empatía, aunque también se las ve como débiles o pasivas, en comparación con los hombres. Debería profundizarse el análisis de estas cuestiones referentes a los fenómenos de percepción del liderazgo femenino.

\section{REFERENCIAS}

Benze, J. J. \& Declerq, E. R. (1985). Content of television spot and female candidates. Journalism Quarterly, 62, 278-283.

Butler, D \& Geis, F. L. (1990). Nonverbal affect responses to male and female leaders: Implications for leadership evaluations. Journal of Personality and Social Psychology, 58, 48-59.

Calvo, P. (2006, 20 de agosto). Ellas avanzan, pero faltan 30 años para la igualdad. Clarín, pp. 32-34.

Casas X., (2010). Hay más jefas pero pocas llegan a ser CEO. Recuperado el 21 de marzo de 2011 http://www. iae.edu.ar/iaehoy/Documents/NG_20100308_Cronista_Debeljuh_MujeresLideres.pdf

Castro Solano, A. (2006). Teorías implícitas del liderazgo, contexto y capacidad de conducción. Anales de psicología, 22 (1), 89-97.

Castro Solano, A. \& Nader, M. (2004). Estilos de liderazgo, contexto y cultura organizacional percibida. Un estudio comparativo en población civil y militar. Boletín de Psicología, 82, 45-63.

Castro Solano, A. \& Lupano Perugini, M.L. (2005). Diferencias individuales en las teorías implícitas del liderazgo y la cultura organizacional percibida. Boletín de Psicología, 85, 89-109.

Carroll, S. J. (1985). Women as Candidate in American Politics. Bloomington: Indiana University Press.

Cheung, F. \& Halpern, D. (2010). Women at the top. Powerful leaders define success as work + family in a culture of gender. American Psychologist, 65 (3), 182-193.

Cohen, J. (1991). Statistical Power Analysis for the Behavioral Sciences. New Jersey: Hillsdale Lawrence Erlbaum Assoc.

Cuadrado, I. (2004). Valores y rasgos estereotípicos de género de mujeres líderes. Psicothema, 16 (2), 270- 275.

Cuadrado, I. Navas, M. \& Molero, F. (2004). El acceso de las mujeres a puestos directivos: género, contexto organizacional y estilos de liderazgo. Rev. de Psicol. Gral. y Aplic., 57 (2), 181-192. 
Cuadrado, I. Navas, M. \& Molero, F. (2006). Mujeres y Liderazgo. Claves Psicosociales del Techo de Cristal. Madrid: Sanz y Torres.

D’Adamo, O., García Beaudoux, V., Ferrari, G. \& Slavinsky, G. (2008). Mujeres candidatas: percepción pública del liderazgo femenino. Revista de Psicología Social, 23 (1), 91-104.

Darcy, R.; Welch, S. \& Clark, J. (1987). Women, electronics, and representation. Nueva York: Longmans.

Diez Gutierrez, E.J.; Terrón Bañuelos, E.; Valle Flórez, R.E. \& Centeno Suárez, B. (2002). Las mujeres y el poder en las organizaciones educativas. Revista Complutense de educación, 13 (2), 485-513.

Druskat, V.U (1994). Gender and leadership style: transformational and transactional leadership in the Roman Catholic Church. Leadership Quarterly, 5 (2), 99-119.

Eagly, A. H. (1987). Sex differences in social behaviour: A social- role interpretation. Hillsdale, $\mathrm{NJ}$ : Lawrence Erbaum.

Eagly, A. H., \& Johonson, B. T. (1990). Gender and leadership style: A meta-analysis. Psychological Bulletin, 108, 233-256.

Eagly, A. H. \& Karau, S. J., (2002). Role congruity theory of prejudice toward female leaders. Psychological Review, 109, 573- 598.

Eagly, A. H.\& Carli L. (2007). Trough the labyrinth. The truth about how women become leaders. Boston: Harvard Business School Press.

Federal Glass Ceiling Commission. (1995). Good for business: Making full use of the nation's human capital: The environment scan: A fact finding report of the Federal Glass Ceiling Commission. Washington, DC: U.S. Government Printing Office.

Gardiner, M. \& Tiggerman, M. (1999). Gender differences in leadership style, job stress, and mental health in male and female dominated industries. Journal of Occupational and Organizational Psychology, 72, 801- 816.

Genovese, M. (1993). Women as national leaders. Newbury Park: Sage.

Glazer-Raymo, J. (1999). Shattering the myths. Women in academe. Baltimore: The Johns Hopkins University Press.

House, R. J., Hanges, P. J, Ruiz -Quintanilla, S. A., Dorfan, P. W., Javidan, M., Dickson, M. et al (1999). Cultural influences on leadership and organisations: Project GLOBE. En W. H. Mobley (Ed.), Advances in global leadership (Vol. 1, pp. 171-233). Stamford, CT: JAI Press.

House, R.J., Javidan, M. \& Dorfman, P.W. (2001). Project GLOBE: An introduction. Applied Psychology: An introduction, 50 (4), 489-505.
Kenney, R. A., Schwartz-Kenney, B. M. \& Blascovich, J. (1996). Implicit Leadership Theories: Defining leaders described as worthy of influence. Personality and Social Psychology Bulletin, 22, 1128 - 1142.

Lord, R., Foti, R. \& De Vader, C. (1984). A test of leadership categorization theory: Internal structure, information processing and leadership perceptions. Organizational behaviour and Human Performance, 34, 343-378.

Lupano Perugini, M.L. \& Castro Solano, A. (2010). Actitudes desfavorables hacia mujeres líderes. Un instrumento para su evaluación. Manuscrito enviado para su evaluación.

Lupano Perugini, M.L. \& Castro Solano A., (2008a). Liderazgo y género. Identificación de Prototipos de liderazgo efectivo. Perspectivas en Psicología, 5 (1), 69-77.

Lupano Perugini, M.L. \& Castro Solano A., (2008b). Prototipos de líderes varones y mujeres. Un análisis comparativo según sexo y puesto ocupado. Acta Psiquiátrica y Psicológica de América Latina, 54 (3), 160-170.

Lupano Perugini, M. L., Castro Solano, A. \& Casullo M.M. (2008). Prototipos de liderazgo masculino y femenino en población militar. Revista de Psicología (PUCP), 25 (2), 195-218.

Morales, J. F. \& Cuadrado, I. (2004). Introducción: Teoría de congruencia de rol del prejuicio hacia líderes femeninos. Rev. de Psicol. Gral. y Aplic., 57 (2), 135- 146.

Morrison, A. M., White, R.P, \& Van Velsor, E. (1987). Breaking the glass ceiling: Can women reach the top of America's largest corporations? Reading, MA: Addison- Wesley.

Sánchez Moreno, M \& López Yáñez, J. (2008). Poder y liderazgo de mujeres responsables de Instituciones Universitarias. Revista Española de Pedagogía, LXVI (240), 345-364.

Ridgeway, C. (2001). Gender, status and leadership. Journal of Social Issues, 57, 637- 655.

Rosener, J. B. (1990). Ways women leads. Harvard Business Review, 68, 119-125.

Ropers-Huilman, B. (2003). Gendered features in higher education. Critical perspective for change. New York: State University of NY Press.

Uriarte, E. \& Ruiz, C. (1999). Mujeres y hombres en las elites políticas españolas: ¿Diferencias o similitudes? REIS, 88, 207-232

Van Engen, M. L.; Van der Leeden R. \& Willemsen, T.M. (2001). Gender, context and leadership styles: A field study. Journal of Occupational and Organizational Psychology, 74, 581-598.

Wofford, J., Godwin, V., \& Wittington, J. (1998). A field study of a cognitive approach to understanding transformational and transaccional leadership. Leadership Quaterly. 9(1), 55-84.

Para citar este artículo:

Lupano Perugino, M. L. \& Castro Solano, A. (2011). Teorías implícitas del liderazgo masculino y femenino según ámbito de desempeño. Ciencias Psicológicas V (2): 139-150. 